\title{
Psychometric Properties and Overlap of the GSQ and AQ among Japanese University Students
}

\author{
${\text { Yuta } U_{j i e}{ }^{1} \& \text { Akio Wakabayashi }}^{2}$ \\ ${ }^{1}$ Graduate School of Advanced Integration Science, Chiba University, Chiba, Japan \\ ${ }^{2}$ Faculty of Letters, Chiba University, Chiba, Japan \\ Correspondence: Yuta Ujie, Graduate School of Advanced Integration Science, Chiba University, 1-33 \\ Yayoi-cho, Inage, Chiba 263-8522, Japan. Tel: 81-432-511-111. E-mail: chiba_psyc_individual@yahoo.co.jp
}

Received: April 3, 2015

doi:10.5539/ijps.v7n2p195
Accepted: April 14, 2015

URL: http://dx.doi.org/10.5539/ijps.v7n2p195

Online Published: May 29, 2015

\begin{abstract}
Individuals with Autism Spectrum Disorders (ASD) have sensory processing problems, and this has recently been included as a core symptom of ASD in DSM-5. The Glasgow Sensory Questionnaire (GSQ), which is based on a dimensional model of ASD, measures the experience of sensory difficulties in daily life. However, the psychometric properties of the GSQ have not been studied in the general population. In this study, we investigated the psychometric properties of the GSQ, including reliability, score distribution, item analysis, gender differences, and the correlation between the Autism Quotient (AQ) and GSQ in a sample of 417 (206 males and 211 females) university students. We also examined the overlap between the AQ and GSQ using a joint factor analysis. The results revealed that the Japanese version of the GSQ has relatively poor psychometric properties compared to the AQ. Gender differences were found in both scales. Individuals with high AQ scores reported experiencing abnormal sensory events more frequently than those with low AQ scores. The overlap between the scales was small; therefore, it might be possible to investigate abnormal sensory processing independently of other symptoms of ASD in the general population.
\end{abstract}

Keywords: Glasgow sensory questionnaire, Autism spectrum quotient, join factor analysis

\section{Introduction}

Autism Spectrum Disorder (ASD) has been largely defined in terms of difficulties in social interaction and communication, patterns of repetitive behavior, narrow interests, and difficulties with sensory processing (Diagnostic and Statistical Manual of Mental Disorders-5: DSM-5; American Psychiatric Association, 2013). These symptoms have been investigated in a number of studies, and the investigation of sensory processing in ASD has gained increasing interest recently. Behavioral and neuropsychological data have suggested that individuals with ASD show atypical input in the visual (Simmons et al., 2009), auditory (Haesen, Boets, \& Wagemans, 2011; O'Connor, 2012), tactile (Foss-Feig, Heacock, \& Cascio, 2012), and vestibular (Baker, Lane, Angley, \& Young, 2008) domains. Some studies have found that more than $90 \%$ of individuals with ASD have a sensory problem, described in terms of either hyper- or hyposensitivity in at least one domain (Crane, Goddard, \& Pring, 2009; Tomchek \& Dunn, 2007).

From a dimensional approach, the degree of autistic-like traits is assumed to be distributed on a continuum over the general population, not only in clinical groups (Baron-Cohen, 1995; Frith, 1991). Based on the dimensional ASD model, Baron-Cohen, Wheelwright, Skinner, Martin, and Clubley (2001) developed the Autism spectrum Quotient (AQ), which is a self-report questionnaire used to assess autistic traits. Its validity to distinguish between clinical and control groups and its test reliability were confirmed in the original (UK) sample (Baron-Cohen et al., 2001), and these findings were replicated in the Netherlands (Hoekstra, Bartels, Cath, \& Boomsma, 2008), Australia (Lau, Kelly, \& Peterson, 2013), and Japan (Wakabayashi, Baron-Cohen, Wheelwright, \& Tojo, 2006). The AQ was designed to assess autistic traits in five domains: social skills, communication, attention to details, attention switching, and imagination. However, this measure does not assess sensory processing problems because it was developed before this feature was included as a core symptom of ASD (Baron-Cohen et al., 2001). 
As a tool to assess sensory problems, the Sensory Profile (SP) (Dunn \& Westman, 1996) has been widely used. This measure was designed to represent children's difficulties with sensory processing, and it is completed by the caregiver. For adults, the Adult/Adolescent Sensory Profile (AASP) (Brown \& Dunn, 2002), which was based on the SP, is typically used. This scale is a 60-item self-report questionnaire that was designed to assess four sensory quadrants: low registration, sensation seeking, sensory sensitivity, and sensation avoidance. There was some evidence that individuals with ASD score higher than controls on the SP (Tomchek \& Dunn, 2007) and AASP (Crane et al., 2009); however, these scales are not well able to assess the degree of hyper- or hyposensitivity in each sensory domain separately.

To address this issue, Robertson and Simmons (2013) developed the Glasgow Sensory Questionnaire (GSQ), which is a self-report questionnaire to assess the frequency of experiencing hyper- or hypo-sensitivity in seven sensory domains: visual, auditory, gustatory, olfactory, tactile, vestibular, and proprioceptive. The original study of the GSQ showed a significant positive correlation between GSQ and AQ scores in the general population (Robertson \& Simmons, 2013). In addition, a score difference between individuals with ASD and those without ASD was found (Horder, Wilson, Mendez, \& Murphy, 2014). Furthermore, Horder et al. (2014) compared the correlation between scores of the AQ, GSQ, and AASP, and revealed that the correlation between the AQ and GSQ was the strongest. These results provided evidence that the GSQ is a more adequate measure of sensory processing problems in ASD.

Although the Japanese version of the GSQ (Takayama et al., 2014) has been developed, its psychometric properties have not been studied adequately, compared to the Japanese version of the AQ (Wakabayashi et al., 2006). For the Japanese version of the AQ, in addition to assessing its validity as a screening tool, item analysis and an examination of its overlap with other scales have been reported (Wakabayashi, Baron-Cohen, \& Ashwin, 2012; Wakabayashi, Baron-Cohen, \& Wheelwright, 2006; Wakabayashi et al., 2006). For the Japanese version of the GSQ, such analyses have not been reported, except for the score difference between a clinical ASD group and controls and a significant positive correlation between the GSQ and AQ (Takayama et al., 2014). Thus, the first aim of this study was to analyze the psychometric properties of the Japanese version of the GSQ, including item analysis and reliability tests of its subscales (seven sensory domains).

In addition, we investigated the overlap between the AQ and the GSQ. The core symptoms of ASD, which were defined in the DSM-5 (American Psychiatric Association, 2013), have been shown in other clinical groups besides those with ASD. For instance, repetitive behavior is seen in both obsessive-compulsive disorder (OCD) and ASD (Russell, Mataix-Cols, Anson, \& Murphy, 2005). Wakabayashi, Baron-Cohen, and Ashwin (2012) reported that, although autistic traits were related to obsessive-compulsive traits as measured by questionnaires, the overlap between the traits was small among the general population. Likewise, sensory processing difficulties have been seen in other disorders, not only in ASD. Some studies showed that those with Sensory Modulation Disorder (SMD) have difficulty responding to sensory input, in the absence of an ASD diagnosis (Miller, Anzalone, Lane, Cermak, \& Osten, 2007; Schoen, Miller, Brett-Green, \& Nielsen, 2009). Furthermore, Pollock, Metz, and Barabash (2014) reported that difficulties with sensory processing have been seen in dysfunctional elimination syndrome (DES). Indeed, although sensory processing difficulties were included as one of the core symptoms of ASD in the DSM-5 (American Psychiatric Association, 2013), these difficulties are not always needed in order to diagnose ASD. The GSQ consists of items measuring "sensory difficulties in daily life", not the "social behaviors" that are assessed by the AQ. We, therefore, hypothesize that the overlap between the AQ and the GSQ will be small, although there will be a relationship between both scales.

In this study, we first investigated the psychometric properties of the Japanese versions of the GSQ and AQ, including reliability, score distribution, item analysis, gender differences, and the correlation with both scales, among Japanese university students. Next, we examined the overlap in the AQ and GSQ scores using multiple regression and joint factor analysis.

\section{Methods}

\subsection{Participants}

Participants were recruited from an introductory psychology class at Chiba University. The sample consisted of 417 students (206 males and 211 females), all of whom were native Japanese speakers. The mean age was 19.6 years $(\mathrm{SD}=1.09)$. All participants provided informed consent and took part in the study voluntarily.

\subsection{Instruments}

2.2.1 Japanese Version of the Glasgow Sensory Questionnaire 
We used the Japanese version of the GSQ (Takayama et al., 2014). This questionnaire contains 42 items on a 5-point Likert-scale: "never", "rarely", "sometimes", "often" and "always." Each response is scored from 0 to 4 points; for example, an item was given 4 points if a participant responded "always" to it. The total possible GSQ scores ranged from 0 to 168 .

\subsubsection{Measures and Covariates}

We used the Japanese version of the AQ (Wakabayashi et al., 2006). This questionnaire consists of 50 items on a 4-point Likert-scale: "definitely disagree", "slightly disagree", "slightly agree" and "definitely agree." According to Austin's scoring method (Austin, 2005), each item was scored from 1 ("definitely disagree") to 4 ("definitely agree") points, and reverse scoring was completed as necessary. The total possible scores ranged from 50 to 200 .

\subsection{Procedure}

Participants completed questionnaires in a group setting during their introductory psychology class. First, 504 university students took the AQ; one month later, 446 students took the GSQ. The results from the students who did not complete both the AQ and GSQ were excluded. In addition, the data from the questionnaires with no responses to multiple items or multiple responses to the same item were excluded. In total, the data from 417 students were analyzed.

\subsection{Data analysis}

Statistical analysis was conducted using R version 2.15.2 for Windows (R Foundation for Statistical Computing, Vienna, Austria). Item analysis of the GSQ was performed using Item-total correlation analysis and Good-Poor analysis for each item. The relationship between the GSQ and AQ was analyzed using Pearson's correlation coefficient. In addition, to examine the overlap between autistic traits and the frequency of abnormal sensory experiences, a multiple regression analysis and joint factor analysis of the GSQ and AQ were conducted. Gender differences and group differences in the AQ sub-groups (high AQ $\geq$ mean AQ + $1 \mathrm{SD}$; low AQ $\leq$ mean AQ - 1 SD) were analyzed using independent sample t-tests.

\section{Results}

\subsection{Psychometric Properties of the GSQ and AQ}

\subsubsection{Reliability}

The participants' mean GSQ and AQ scores are shown in Table 1. The GSQ kurtosis and skewness were 1.196 and .351, respectively, and the kurtosis and skewness of the AQ were .275 and .198 , respectively. The Kolmogorov-Smirnov test revealed that both the GSQ and AQ scores were normally distributed $(p>.05)$.

The internal consistency (Cronbach's alpha) of the GSQ was .84, which was an acceptable level of reliability. Similarly, the scores of the AQ indicated acceptable reliability (Cronbach's alpha was .84). The Cronbach's alphas for the subscales of the GSQ and AQ are shown in Table 1.

Table 1. Mean, SD, Cronbach's $\alpha$, and gender differences for each subscale of the AQ and GSQ

\begin{tabular}{lllllllll}
\hline & & \multicolumn{2}{c}{$\begin{array}{l}\text { All } \\
(\mathrm{N}=417)\end{array}$} & \multicolumn{2}{l}{$\begin{array}{l}\text { Males } \\
(\mathrm{n}=206)\end{array}$} & $\begin{array}{l}\text { Females } \\
(\mathrm{n}=211)\end{array}$ & $\begin{array}{l}\text { Gender } \\
\text { differences }\end{array}$ \\
\hline & Cronbach's $\alpha$ & Mean & SD & Mean & SD & Mean & SD & t \\
AQ total & .84 & 118.0 & 13.43 & 120.1 & 13.81 & 115.7 & 12.71 & $3.34^{* *}$ \\
Social skills & .80 & 23.1 & 5.18 & 23.7 & 5.47 & 22.4 & 4.81 & $2.54^{* *}$ \\
Attention switching & .50 & 26.1 & 3.69 & 26.3 & 3.62 & 25.8 & 3.76 & 1.24 \\
Attention to details & .61 & 24.6 & 4.31 & 24.6 & 4.35 & 24.5 & 4.27 & .18 \\
Communication & .66 & 22.4 & 4.15 & 23.0 & 4.17 & 21.9 & 4.06 & $2.72^{* *}$ \\
Imagination & .59 & 21.7 & 3.87 & 22.5 & 3.99 & 21.0 & 3.63 & $3.86^{* *}$ \\
GSQ total & .84 & 57.6 & 15.29 & 59.8 & 16.32 & 55.5 & 13.91 & $2.92^{* *}$ \\
Visual & .51 & 10.2 & 3.91 & 10.4 & 4.13 & 10.0 & 3.68 & .97 \\
Auditory & .56 & 13.6 & 3.37 & 13.9 & 3.39 & 13.3 & 3.33 & 1.90 \\
\hline
\end{tabular}




\begin{tabular}{lllllllll}
\hline Gustatory & .43 & 6.3 & 2.84 & 6.6 & 3.06 & 6.0 & 2.58 & $2.22^{*}$ \\
Olfactory & .42 & 6.9 & 2.73 & 7.3 & 2.94 & 6.5 & 2.44 & $3.11^{* *}$ \\
Tactile & .31 & 6.6 & 2.85 & 7.2 & 2.98 & 6.0 & 2.59 & $4.41^{* *}$ \\
Vestibular & .52 & 7.0 & 3.37 & 7.1 & 3.52 & 6.9 & 3.22 & .77 \\
Proprioceptive & .49 & 7.0 & 3.05 & 7.3 & 3.27 & 6.8 & 2.81 & 1.48 \\
\hline
\end{tabular}

Note. ${ }^{*} \mathrm{p}<.05,{ }^{* *} \mathrm{p}<.01$

\subsubsection{Item Analysis of the GSQ}

The results of the item-total correlation and good-poor analysis for each item of the GSQ are shown in Table 2. Item-total correlation analysis revealed that all 42 items had significant positive correlations with the total GSQ $(p<.05)$, although the results on two items (Items 17 and 36) were slightly lower $(\mathrm{r}<.20)$. Good-poor analysis showed that high scorers on the total GSQ scored significantly higher $(p<.05)$ than did low scorers on all items except one (Item 22). These results suggest that Items 17, 22, and 36 were not adequate to assess the frequency of abnormal sensory experiences among the general population. However, these items might possibly reflect abnormal sensory experiences found only in high-scoring individuals on the AQ and individuals with ASD; therefore, these items were retained in the following analysis.

Table 2. Results of good-poor analysis and item-total correlations

\begin{tabular}{|c|c|c|c|c|}
\hline Items & Mean & SD & $t$ & Item-total correlations \\
\hline \multicolumn{5}{|l|}{ Visual } \\
\hline 4 & 1.7 & 1.16 & $7.63^{* * *}$ & .41 \\
\hline 8 & 1.8 & 1.09 & $5.80^{* * *}$ & .31 \\
\hline 11 & .7 & .90 & $5.21 * * *$ & .26 \\
\hline 18 & 1.2 & .95 & $6.23^{* * *}$ & .41 \\
\hline 19 & 2.0 & 1.17 & $6.39 * * *$ & .42 \\
\hline 42 & 1.0 & 1.09 & $8.21 * * *$ & .46 \\
\hline \multicolumn{5}{|c|}{ Auditory } \\
\hline 6 & 2.2 & .96 & $6.38^{* * *}$ & .38 \\
\hline 9 & 2.3 & 1.05 & $5.32 * * *$ & .38 \\
\hline 14 & 2.3 & .91 & $7.94 * * *$ & .39 \\
\hline 25 & 2.7 & 1.03 & $4.93^{* * *}$ & .29 \\
\hline 31 & 2.6 & .96 & $7.06^{* * *}$ & .40 \\
\hline 33 & 1.5 & 1.13 & $6.65 * * *$ & .47 \\
\hline \multicolumn{5}{|c|}{ Gustatory } \\
\hline 2 & 1.5 & 1.00 & $4.44^{* * *}$ & .26 \\
\hline 23 & 1.7 & 1.02 & $6.56^{* * *}$ & .38 \\
\hline 25 & 2.7 & 1.03 & $4.93 * * *$ & .29 \\
\hline 28 & .7 & .82 & $4.54 * * *$ & .38 \\
\hline 35 & 6 & .90 & $6.80^{* * *}$ & .41 \\
\hline 39 & 1.1 & .98 & $7.19^{* * *}$ & .39 \\
\hline \multicolumn{5}{|c|}{ Olfactory } \\
\hline 7 & 1.8 & .94 & $5.13^{* * *}$ & .35 \\
\hline
\end{tabular}




\begin{tabular}{|c|c|c|c|c|}
\hline 13 & 1.9 & 1.02 & $8.85^{* * *}$ & .43 \\
\hline 17 & .2 & .57 & $2.22 *$ & .17 \\
\hline 21 & 1.9 & 1.27 & $6.55^{* * *}$ & .32 \\
\hline 24 & .7 & .93 & $6.90 * * *$ & .42 \\
\hline 36 & .5 & .81 & $2.12 *$ & .16 \\
\hline \multicolumn{5}{|c|}{ Tactile } \\
\hline 1 & 1.2 & 1.12 & $4.15^{* * *}$ & .20 \\
\hline 15 & .7 & 1.00 & $4.84 * * *$ & .34 \\
\hline 16 & 1.3 & .92 & $5.95^{* * *}$ & .33 \\
\hline 22 & .7 & 1.04 & $1.73 \dagger$ & .21 \\
\hline 27 & .7 & .88 & $5.44 * * *$ & .41 \\
\hline 40 & 2.0 & 1.05 & $6.02 * * *$ & .37 \\
\hline \multicolumn{5}{|c|}{ Vestibular } \\
\hline 10 & 1.4 & 1.12 & $5.08 * * *$ & .35 \\
\hline 12 & 1.1 & 1.07 & $7.11 * * *$ & .38 \\
\hline 20 & 1.4 & 1.02 & $7.40^{* * *}$ & .41 \\
\hline 30 & .7 & .97 & $6.95^{* * *}$ & .45 \\
\hline 32 & 1.4 & 1.15 & $4.65^{* * *}$ & .29 \\
\hline 34 & 1.0 & .92 & $7.10^{* * *}$ & .45 \\
\hline \multicolumn{5}{|c|}{ Proprioceptive } \\
\hline 3 & 1.3 & .99 & $4.94 * * *$ & .28 \\
\hline 5 & 1.4 & 1.04 & $3.54^{* * *}$ & .25 \\
\hline 29 & 1.1 & .84 & $5.70 * * *$ & .37 \\
\hline 36 & .5 & .81 & $2.12 *$ & .16 \\
\hline 37 & 1.6 & 1.01 & $8.45^{* * *}$ & .48 \\
\hline 41 & .8 & .90 & $7.01 * * *$ & .41 \\
\hline
\end{tabular}

Note. $p<.10, * p<.05, * * p<.01, * * * p<.001$

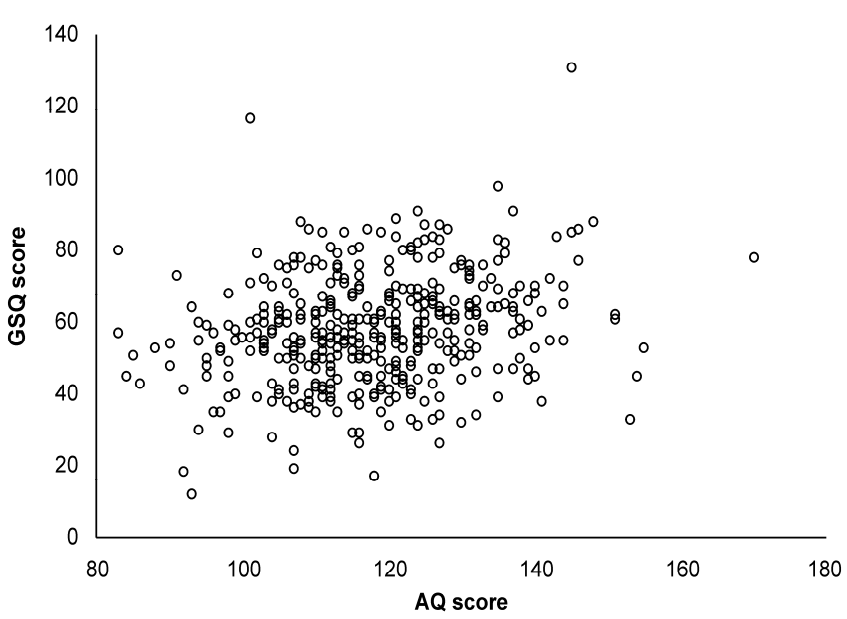

Figure 1. The relationship between GSQ and AQ scores 


\subsubsection{Gender Differences}

As shown in Table 1, the mean total GSQ score was higher for males than for females [ $t(415)=2.92, p<.01]$. On the subscales of the GSQ, males scored significantly higher than did females in three sensory domains: gustatory $[\mathrm{t}(415)=2.22, \mathrm{p}<.05]$, olfactory $[\mathrm{t}(415)=3.11, \mathrm{p}<.01]$, and tactile [ $\mathrm{t}(415)=4.41, \mathrm{p}<.01]$. The total AQ score for males was significantly higher than that for the females $[t(415)=3.34, p<.01]$, and three subscales of the AQ were higher for males than for females: social skills [t $(415)=2.54, \mathrm{p}<.01]$, communication [ $\mathrm{t}(415)=2.72, \mathrm{p}<.01]$, and imagination [ $\mathrm{t}(415)=3.86, \mathrm{p}<.01]$.

\subsection{The Relationship Between the GSQ and AQ}

\subsubsection{Correlations Between the GSQ and AQ}

The relationship between the total GSQ and AQ scores is shown in Figure 1. Pearson's correlation coefficients between total scores on the GSQ and AQ and between their subscales are summarized in Table 3. The total AQ score was positively and significantly correlated with the total GSQ score and all of its subscales. Additionally, there were significant positive correlations between all of the subscales of AQ combined and the total GSQ score. However, only some of the AQ subscales and GSQ were significantly correlated (Table 3).

Table 3. Correlations between the subscales of the GSQ and AQ

\begin{tabular}{|c|c|c|c|c|c|c|}
\hline & AQ total & $\begin{array}{l}\text { Social } \\
\text { skills }\end{array}$ & $\begin{array}{l}\text { Attention } \\
\text { switching }\end{array}$ & $\begin{array}{l}\text { Attention } \\
\text { to details }\end{array}$ & Communication & Imagination \\
\hline GSQ total & $.25 * *$ & $.11 *$ & $.22 * *$ & $.17 * *$ & $.20 * *$ & $.11^{*}$ \\
\hline Visual & $.13 * *$ & .03 & $.10^{*}$ & $.22 * *$ & $.09^{\dagger}$ & -.03 \\
\hline Auditory & $.19 * *$ & $.14^{* *}$ & $.22 * *$ & $.09^{\dagger}$ & $.12 *$ & .03 \\
\hline Gustatory & $.12 *$ & .04 & $.13 * *$ & .05 & $.12 *$ & .05 \\
\hline Olfactory & $.12 *$ & -.02 & .07 & $.13^{* *}$ & $.10^{*}$ & $.11^{*}$ \\
\hline Tactile & $.32 * *$ & $.21 * *$ & $.23 * *$ & $.09^{\dagger}$ & $.22 * *$ & $.26^{* *}$ \\
\hline Vestibular & $.14 * *$ & .02 & $.11 *$ & $.15 * *$ & $.15^{* *}$ & .02 \\
\hline Proprioceptive & $.21 * *$ & $.10^{*}$ & $.19 * *$ & .07 & $.19 * *$ & $.14 * *$ \\
\hline
\end{tabular}

\subsubsection{Group Differences Between Low and High AQ}

To examine whether individuals with high autistic traits report a higher frequency of abnormal sensory experiences, from participants, we picked sixty participants with scoring 132 or more (mean AQ+1 SD), and another sixty participants with scoring 104 or less (mean AQ-1 SD). We regarded the former as a high AQ group and the latter as a low AQ group.The results of the GSQ and AQ for each group are shown in Table 4. The total GSQ score for the high AQ group was significantly higher than for the low AQ [ $t(118)=3.94, p<.01]$. On the subscales of the GSQ, visual [ $\mathrm{t}(118)=2.36, \mathrm{p}<.05$ ], auditory [ $\mathrm{t}(118)=3.38, \mathrm{p}<.01]$, tactile [ $\mathrm{t}(118)=6.11, \mathrm{p}$ $<.01]$, and proprioceptive $[\mathrm{t}(118)=3.50, \mathrm{p}<.01]$ domain scores were significantly higher for the high than for the low AQ group.

Table 4. Group differences between low and high AQ participants

\begin{tabular}{lllllll}
\hline & \multicolumn{2}{l}{ Low AQ $(\mathrm{n}=60)$} & \multicolumn{2}{l}{ High AQ $(\mathrm{n}=60)$} & \multicolumn{2}{c}{ Low AQ vs. High AQ } \\
& Mean & SD & Mean & SD & $t$ & $p$ \\
\hline AQ total & 97.4 & 5.82 & 139.7 & 7.04 & 35.82 & .00 \\
Social skills & 16.7 & 2.98 & 29.6 & 3.76 & 20.78 & .00 \\
Attention switching & 22.5 & 3.62 & 29.9 & 3.16 & 11.87 & .00 \\
\hline
\end{tabular}




\begin{tabular}{lllllll}
\hline Attention to details & 23.2 & 4.28 & 26.5 & 3.89 & 4.33 & .00 \\
Communication & 16.9 & 2.95 & 27.5 & 2.93 & 19.59 & .00 \\
Imagination & 18.0 & 3.29 & 26.3 & 3.31 & 13.75 & .00 \\
GSQ total & 52.9 & 15.8 & 64.6 & 16.77 & 3.94 & .00 \\
Visual & 9.5 & 4.19 & 11.3 & 4.02 & 2.36 & .02 \\
Auditory & 12.7 & 3.63 & 14.8 & 3.22 & 3.38 & .00 \\
Gustatory & 6.1 & 2.96 & 7.0 & 3.36 & 1.50 & .14 \\
Olfactory & 6.6 & 2.85 & 7.3 & 2.86 & 1.37 & .17 \\
Tactile & 5.4 & 2.52 & 8.5 & 2.99 & 6.11 & .00 \\
Vestibular & 6.8 & 3.68 & 7.9 & 3.53 & 1.59 & .11 \\
Proprioceptive & 5.8 & 2.93 & 7.9 & 3.70 & 3.50 & .00 \\
\hline
\end{tabular}

\subsection{The Overlap between Items of the GSQ and $A Q$}

\subsubsection{Multiple Regression Analysis}

To examine whether AQ scores could be accounted for by GSQ scores, we conducted a multiple regression analysis with the subscales of the AQ as predictor variables and total GSQ score as the objective variable. The subscales of the AQ predicted $8.3 \%$ of the variance in the total GSQ score. Similarly, multiple regression analyses were performed with the subscales of the AQ as predictor variables and each sensory domain of the GSQ as objective variables. The results also showed small $\mathrm{R}^{2}$ values ranging from $1.4 \%$ to $9.8 \%$ (visual $=6.1 \%$, auditory $=5.8 \%$, gustatory $=1.4 \%$, olfactory $=3.2 \%$, tactile $=9.8 \%$, vestibular $=4.4 \%$, and proprioceptive $=$ $4.6 \%)$.

\subsubsection{Joint Factor Analysis of the GSQ and AQ}

To examine the degree of overlap between the GSQ and AQ, we conducted a joint factor analysis among their subscales. Similar to the procedure used by Wakabayashi et al. (2006), a principal factor analysis was carried out on the inter-subscale correlation matrix of the GSQ and AQ. As shown in Fig. 2, the eigenvalues of the first two factors were greater than one. Table 5 summarizes the results of the factor pattern matrix by an oblimin-rotated two-factor solution. All of the subscales of the GSQ were loaded onto Factor 1, while four subscales of the AQ were loaded onto Factor 2. Therefore, we interpreted Factor 1 as the frequency of abnormal sensory experiences and Factor 2 as autistic traits. However, "attention to details" did not load on either factor. This finding, that "attention to details" was slightly different from the other four AQ subscales, was in agreement with the results of previous studies [the results of a structural factor model of AQ (Hoekstra et al., 2008) and the results of a joint factor analysis among the AQ and NEO-PI-R (Wakabayashi et al., 2006)].

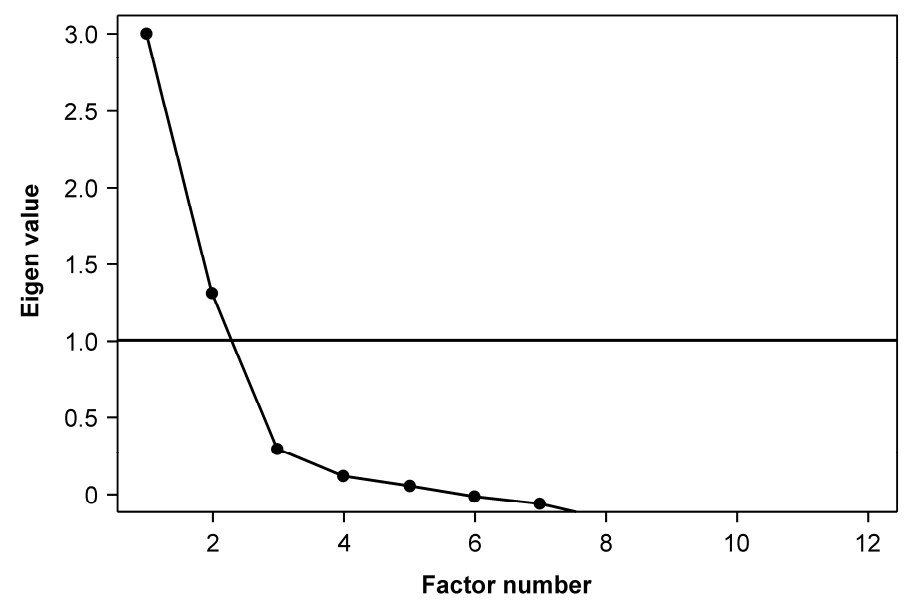

Figure 2. Scree plot of the GSQ and AQ joint factor analysis 
Table 5. Oblimin-rotated two-factor solution for each subscale of the AQ and GSQ

\begin{tabular}{lllll}
\hline & Factor 1 & Factor 2 & $h^{2}$ & $u^{2}$ \\
\hline AQ & & & & \\
Social skills & -.07 & .74 & .52 & .48 \\
Attention switching & .10 & .54 & .33 & .67 \\
Attention to details & .21 & -.13 & .05 & .95 \\
Communication & .04 &. $\mathbf{7 9}$ & .64 & .36 \\
Imagination & -.03 & .63 & .40 & .61 \\
GSQ & & & & \\
Visual & $\mathbf{. 6 1}$ & -.07 & .35 & .65 \\
Auditory & $\mathbf{. 5 9}$ & .03 & .35 & .65 \\
Gustatory & $\mathbf{. 6 8}$ & -.03 & .45 & .55 \\
Olfactory & $\mathbf{. 6 2}$ & -.06 & .37 & .63 \\
Tactile & $\mathbf{. 5 5}$ & .19 & .38 & .62 \\
Vestibular & $\mathbf{. 6 5}$ & -.04 & .42 & .58 \\
Proprioceptive & $\mathbf{. 6 7}$ & .07 & .47 & .53 \\
Variance & .23 & .16 & & \\
\hline
\end{tabular}

Note. Items with loadings above .40 are shown in bold

\section{Discussion}

This study examined the psychometric properties and overlap of the Japanese versions of the GSQ and AQ among Japanese university students. The results showed that both scales have good psychometric properties concerning test reliability and score distribution. In the examination of gender differences, males scored higher than did females on both the GSQ and AQ. For the AQ, such gender differences are expected, based on the sex ratio in diagnosing ASD (Baron-Cohen et al., 2001). The gender difference in the total AQ score was consistent with the result of previous studies (Austin, 2005; Baron-Cohen et al., 2001; Hoekstra et al., 2008; Horder et al., 2014; Robertson \& Simmons, 2013; Stewart \& Austin, 2009; Wakabayashi et al., 2006). For the GSQ, this study is the first to report gender differences. This indicates that the sex ratio in diagnosing ASD reflects on the dimensional model of the GSQ, as well as the AQ.

However, for the psychometric properties of the GSQ, the results of the item analysis revealed that some items were not at an acceptable level. This suggests that some items might be not adequate for measuring sensory difficulties in the general sample, although these items could reflect sensory difficulties in the clinical ASD sample. Furthermore, we were unable to obtain satisfactory reliabilities on the subscales of the GSQ compared to those of the AQ. This suggests that there is not enough reliability to assess the "experience of abnormal events" for each sensory domain independently, at least in the general population. Indeed, the original study of the GSQ (Robertson \& Simmons, 2013) showed that the single-factor GSQ model was appropriate from the result of a principal components analysis. In clinical studies of ASD, although individuals with ASD presented processing difficulty in one or more sensory domains (Baker et al., 2008; Foss-Feig et al., 2012; Simmons et al., 2009; Tomchek \& Dunn, 2007), the symptom of hyper- or hyposensitivity are seen to be common to some extent, regardless of sensory domains. Therefore, the GSQ subscales might be adequately divided into the symptoms (hyper- and hypo-sensitivity), but not into the sensory domains, when necessary.

With regard to the relationship between the AQ and GSQ, as we hypothesized, our results showed a significant correlation but a small overlap between both scales. A significant positive correlation was found, although the size of the correlation in this study was small (Horder et al., 2014; Robertson \& Simmons, 2013; Takayama et al., 2014). We also found differences between the high and low AQ individuals in terms of the total GSQ. These results are partially consistent with a previous study (Robertson \& Simmons, 2013) and suggest that individuals with high autistic traits tend to experience hyper- or hyposensitivity sensory events in their daily lives. On the degree of the overlap between the GSQ and the AQ, however, the result of the multiple regression showed that 
AQ subscales predicted only $8.3 \%$ of the variance in the total GSQ score. Furthermore, the result of joint factor analysis of the GSQ and the AQ revealed a two-factor solution, which suggests that those factors correspond with the GSQ and AQ. Factor 1 consisted of all the GSQ subscales, and Factor 2 consisted of four AQ subscales (with the exception of "attention to details"). This result indicates that the frequency of abnormal sensory experiences on the GSQ can be assessed independently. Thus, we found that, although individuals with high AQ experience more abnormal sensory events in their daily lives than those with low AQ, the overlap between GSQ and AQ items would be small among the general population.

This finding might have important implications for an analog design to study difficulties in sensory processing in a typically developing sample. Early research reported differences in sympathetic nervous system functioning between children with ASD and those with SMD, although both groups of children showed more sensory processing difficulties than controls (Schoen et al., 2009). Such a comparison would be useful in understanding difficulties with sensory processing in ASD. However, further research into such comparisons may be difficult in clinical studies, because it would be difficult to control the severity of symptoms and IQ within or between clinical groups. Given the small overlap between the AQ and GSQ in this study, it is possible that difficulties in sensory processing might be studied by comparing sensory profiles between individuals with high GSQ and high AQ and those with high GSQ but low AQ within the general population. To do this, more research, such as that investigating relationships between the GSQ and sensory performance on experimental tasks, is needed.

\subsection{Limitations and Conclusion}

There are some limitations to our study. First, because we used only AQ and GSQ, the effect of other traits on the GSQ was not clear. Several studies have shown a relationship between anxiety and sensory processing difficulties in ASD (Horder et al., 2014; Mazurek, Keefer, Shui, \& Vasa, 2014). Van Steensel, Bögels, and Perrin (2011) reviewed 31 studies of ASD and revealed that $39.6 \%$ of children with ASD have at least one DSM-IV anxiety disorder. Mizurek et al. (2014) suggested a relationship between anxiety and sensory over-responsivity in children with ASD. In an analog study of ASD, Horder et al. (2014) found a positive correlation between the self-report Spielberger State/Trait Anxiety Inventory and GSQ. Furthermore, a similar relationship was found in the study of other clinical domains, such as children with selective eating (Farrow \& Cloulthard, 2012). In this study, the GSQ scores might possibly reflect the traits of anxiety found among the general population. Therefore, further study is needed to clarify the relationships and overlaps between the AQ, GSQ, and anxiety traits.

Second, the results of the current study might depend on the homogeneity of our sample, which consisted of students recruited from a single university. As it has been shown that the factor structure of the AQ (Austin, 2005; Hoekstra et al., 2008; Stewart \& Austin, 2008) and the correlation between the AQ and the GSQ (Horder et al., 2014; Robertson \& Simmons, 2013; Takayama et al., 2014) varied slightly between a clinical and neurotypical sample, the results of the current study might have been different had the sample reflected a more diverse general population. Further studies are required to investigate these issues.

In conclusion, the Japanese version of the GSQ has relatively poor psychometric properties, compared to the AQ. Gender differences were found for both scales. With regard to the relationship between the AQ and GSQ, individuals with high autistic traits experienced abnormal sensory events with higher frequency than those with low autistic traits in their daily lives. However, the overlap between the scales was small; therefore, it might be possible to investigate abnormal sensory processing independently of other symptoms of ASD (difficulties in social interactions, patterns of repetitive behaviors, and narrow interests) in the general population.

\section{Acknowledgments}

We would like to thank Dr. R. Hashimoto and Y. Takayama for allowing us to use the Japanese version of the Glasgow Sensory Questionnaire (GSQ). This study was supported by a Grant-in-Aid from the Japan Society for the Promotion of Science Fellows (Grant No.26-8144). The Japan Society for the Promotion of Science Fellows had no role in the study design, collection, analysis, or interpretation of the data, and they were not involved in the writing of the report or the decision to submit the article for publication.

The authors declare no conflict of interest.

\section{References}

American Psychiatric Association. (1994). Diagnostic and statistical manual of mental disorders (4th ed.). Washington, DC: American Psychiatric Association.

American Psychiatric Association. (2013). Diagnostic and statistical manual of mental disorders (5th ed.). Washington, DC: American Psychiatric Association. http://dx.doi.org/10.1176/appi.books.9780890425596 
Austin, E. J. (2005). Personality correlates of the broader autism phenotype as assessed by the Autism Spectrum Quotient (AQ). Personality and Individual Differences, 38, 451-460. http://dx.doi.org/10.1016/ j.paid.2004.04.022

Baker, A. E., Lane, A., Angley, M. T., \& Young, R. L. (2008). The relationship between sensory processing patterns and behavioural responsiveness in autistic disorder: A pilot study. Journal of Autism and Developmental Disorders, 38(5), 867-875. http://dx.doi.org/10.1007/s10803-007-0459-0

Baron-Cohen, S. (1995). Mindblindness: An essay on autism and theory of mind. Boston, MA: MIT Press/Bradford Books.

Baron-Cohen, S., Wheelwright, S., Skinner, R., Martin, J., \& Clubley, E. (2001). The Autism-Spectrum Quotient (AQ): Evidence from Asperger syndrome/high-functioning autism, males and females, scientists and mathematicians. Journal of Autism and Developmental Disorders, 31, 5-17. http://dx.doi.org/10.1023/ A:1005653411471

Crane, L., Goddard, L., \& Pring, L. (2009). Sensory processing in adults with autism spectrum disorders. Autism, 13, 215-228. http://dx.doi.org/10.1177/1362361309103794

Dunn, W., \& Westman, K. (1997). The sensory profile: The performance of a national sample of children without disabilities. American Journal of Occupational Therapy, 51, 25-34. http://dx.doi.org/10.5014/ ajot.51.1.25

Farrow, C. V., \& Cloulthard, H. (2012). Relationships between sensory sensitivity, anxiety and selective eating in children. Appetite, 58, 842-846. http://dx.doi.org/10.1016/j.appet.2012.01.017

Foss-Feig, J. H., Heacock, J. L., \& Cascio, C. J. (2012). Tactile responsiveness patterns and their association with core feature in autism spectrum disorders. Research in Autism Spectrum Disorders, 6, 337-344. http://dx.doi.org/10.1016/j.rasd.2011.06.007

Frith, U. (1991). Autism and Asperger's Syndrome. Cambridge: Cambridge University Press. http://dx.doi.org/ $10.1017 / \mathrm{CBO} 9780511526770$

Haesen, B., Boets, B., \& Wagemans, J. (2011). A review of behavioral and electrophysiological studies on auditory processing and speech perception in autism spectrum disorders. Research in Autism Spectrum Disorders, 5, 701-714. http://dx.doi.org/10.1016/j.rasd.2010.11.006

Happe, F., \& Frith, U. (2006). The weak coherence account: Detail-focused cognitive style in Autism Spectrum Disorders. Journal of Autism and Developmental Disorders, 36(1), 5-25. http://dx.doi.org/10.1007/ s10803-005-0039-0

Hoekstra, R. A., Bartels, M., Cath, D. C., \& Boomsma, D. I. (2008). Factor structure, reliability and criterion validity of the Autism-Spectrum Quotient (AQ): A study in Dutch population and patient groups. Journal of Autism and Developmental Disorders, 38, 1555-1566. http://dx.doi.org/10.1007/s10803-008-0538-x

Horder, J., Wilson, C. E., Mendez, M. A., \& Murphy, D. G. (2014). Autistic traits and abnormal sensory experiences in adults. Journal of Autism and Developmental Disorders, 44, 1461-1469. http://dx.doi.org/10.1007/s10803-013-2012-7

Lau, W. Y. P., Kelly, A. B., \& Peterson, C. C. (2013). Further evidence on the factorial structure of the autism spectrum quotient (AQ) for adults with and without a clinical diagnosis of autism. Journal of Autism and Developmental Disorders, 43, 2807-2815. http://dx.doi.org/10.1007/s10803-013-1827-6

Mazurek, M. O., Keefer, A., Shui, A., \& Vasa, R. A. (2014). One-year course and predictors of abdominal pain in children with autism spectrum disorders: The role of anxiety and sensory over-responsivity. Research in Autism Spectrum Disorders, 8, 1508-1515. http://dx.doi.org/10.1016/j.rasd.2014.07.018

Miller, L. J., Anzalone, M. E., Lane, S. J., Cermak, S. A., \& Osten, E. T. (2007). Concept evolution in sensory integration: A proposed nosology for diagnosis. American Journal of Occupational Therapy, 61, 135-140. http://dx.doi.org/10.5014/ajot.61.2.135

O'Connor, K. (2012). Auditory processing in autism spectrum disorder: A review. Neuroscience and Biobehavioral Reviews, 36, 836-854. http://dx.doi.org/10.1016/j.neubiorev.2011.11.008

Pollock, M. R., Metz, A. E., \& Barabash, T. (2014). Brief Report-Association between dysfunctional elimination syndrome and sensory processing disorder. American Journal of Occupational Therapy, 68, 472-477. http://dx.doi.org/10.5014/ajot.2014.011411 
Robertson, A. E., \& Simmons, D. R. (2012). The relationship between sensory sensitivity and autistic traits in the general population. Journal of Autism and Developmental Disorders, 43(4), 775-784. http://dx.doi.org/ 10.1007/s10803-012-1608-7

Russell, A. J., Mataix-Cols, D., Anson, M., \& Murphy, D. G. M. (2005). Obsessions and compulsions in Asperger syndrome and high-functioning autism. British Journal of Psychiatry, 186, 525-528. http://dx.doi.org/10.1192/bjp.186.6.525

Schoen, S. A., Miller, L. J., Brett-Green, B. A., \& Nielsen, D. M. (2009). Physiological and behavioral differences in sensory processing: A comparison of children with Autism Spectrum Disorder and Sensory Modulation Disorder. Frontiers in Integrative Neuroscience, 3, 29. http://dx.doi.org/10.3389/ neuro.07.029.2009

Simmons, D. R., Robertson, A. E., McKay, L. S., Toal, E., McAleer, P., \& Pollick, F. E. (2009). Vision in autism spectrum disorders. Vision Research, 49, 2705-2739. http://dx.doi.org/10.1016/j.visres.2009.08.005

Stewart, M., \& Austin, E. J. (2009). The structure of the autism-spectrum quotient (AQ): Evidence from a student sample in Scotland. Personality and Individual Differences, 47(3), 224-228. http://dx.doi.org/ 10.1016/j.paid.2009.03.004

Takayama, Y., Hashimoto, R., Tani, M., Kanai, C., Yamada, T., Watanabe, H., ... \& Iwanami, A. (2014). Standardization of the Japanese version of the Glasgow Sensory Questionnaire (GSQ). Research in Autism Spectrum Disorders, 8, 347-353. http://dx.doi.org/10.1016/j.rasd.2013.12.017

Tomchek, S. D., \& Dunn, W. (2007). Sensory processing in children with and without Autism: A comparative study using the Short Sensory Profile. American Journal of Occupational Therapy, 61, 190-200. http://dx.doi.org/10.5014/ajot.61.2.190

Van Steensel, F. J. A., Bögels, S. M., \& Perrin, S. (2011). Anxiety disorders in children and adolescents with autistic spectrum disorders: A meta-analysis. Clinical Child and Family Psychological Review, 3, 302-317. http://dx.doi.org/10.1007/s10567-011-0097-0

Wakabayashi, A., Baron-Cohen, S., \& Ashwin, C. (2012). Do the traits of autism-spectrum overlap with those of schizophrenia or obsessive-compulsive disorder in the general population? Research in Autism Spectrum Disorders, 6, 717-725. http://dx.doi.org/10.1016/j.rasd.2011.09.008

Wakabayashi, A., Baron-Cohen, S., \& Wheelwright, S. (2006). Are autistic traits an independent personality dimension? A study of the Autism-Spectrum Quotient (AQ) and the NEO-PI-R. Personality and Individual Differences, 41, 873-883. http://dx.doi.org/10.1016/j.paid.2006.04.003

Wakabayashi, A., Baron-Cohen, S., Wheelwright, S., \& Tojo, Y. (2006). The Autism-Spectrum Quotient (AQ) in Japan: A crosscultural comparison. Journal of Autism and Developmental Disorders, 36, 263-270. http://dx.doi.org/10.1007/s10803-005-0061-2

Watling, R. L., Deitz, J., \& White, O. (2001). Comparison of sensory profile scores of young children with and without autism spectrum disorders. American Journal of Occupational Therapy, 55(4), 416-423. http://dx.doi.org/10.5014/ajot.55.4.416

\section{Copyrights}

The copyright for this article is retained by the authors, with first publication rights granted to the journal.

This is an open-access article distributed under the terms and conditions of the Creative Commons Attribution license (http://creativecommons.org/licenses/by/3.0/). 